\title{
Transapical beating heart cardioscopy technique for off-pump visualization of heart valves
}

\author{
Muralidhar Padala, $\mathrm{PhD},{ }^{\mathrm{a}}$ Jorge H. Jimenez, $\mathrm{PhD},{ }^{\mathrm{b}}$ Ajit P. Yoganathan, $\mathrm{PhD},{ }^{\mathrm{b}}$ Albert Chin, $\mathrm{MD},{ }^{\mathrm{c}}$ and \\ Vinod H. Thourani, MD
}

Objective: Endoscopic methods to perform intracardiac procedures are of enormous interest, with the introduction of transcatheter techniques for complex cardiac procedures. In the present study, we demonstrate the use of a novel transapical cardioscopy system to visualize intracardiac structures in a porcine model.

Methods: The cardioscope was designed to mount a miniature CCD camera at its tip and was covered in a blunt convex Plexiglass top that allowed displacement and visualization of the tissue in front of the cardioscope. Transapical access for 11-mm cardioscopy was performed by way of a median sternotomy $(n=4)$ and minithoracotomy $(\mathrm{n}=1)$ in an anesthetized porcine model, and various cardiac structures were imaged under beating heart conditions. The images from the camera were projected onto a monitor for the operator to guide cardioscope positioning.

Results: Video images and identification of structures on the left side of an in vivo beating porcine heart were obtained. Initially, the papillary muscle and mitral valve components were evaluated. The left atrium was entered, and the pulmonary vein orifices and atrial appendage were confirmed. Next, the camera was positioned within the left ventricle, and the ventricular portion of the trileaflet aortic valve was inspected. Using direct visualization, the camera was passed into the proximal ascending aorta. The left and right coronary arteries were also visualized. A catheter was introduced by way of a side port to confirm the position of the aortic valve leaflets during visualization. The pig experienced no significant decrease in blood pressure and maintained a stable heart rate throughout the procedure. The port was removed, and the transapical incision was closed with minimal blood loss during the procedure and closure of the orifice.

Conclusions: Transapical cardioscopy is a novel approach that allows for precise visualization of intracardiac structures within a beating porcine heart without the use of cardiopulmonary bypass. This technique might allow for more successful minimally invasive valvular, intracardiac, or ascending aortic procedures without the use of radiation. (J Thorac Cardiovasc Surg 2012;144:231-4)

Endoscopic methods to perform intracardiac procedures are of enormous interest, with the introduction of transcatheter techniques for complex cardiac procedures. To introduce catheters and maneuver them to the desired location in the heart, echocardiographic or angiographic guidance is necessary. An inherent drawback of these modalities is that the field of view is often a 2-dimensional plane, which might be significantly different from the 3-dimensional space through which the catheter tip needs maneuvering. Although advances in 3-dimensional echocardiography have significantly improved imaging, they still do not provide the frontal view of the catheter tip to assess the tissue

From the Division of Cardiothoracic Surgery, ${ }^{\mathrm{a}}$ Emory University School of Medicine, Atlanta, Ga; Department of Biomedical Engineering, ${ }^{\mathrm{b}}$ Georgia Institute of Technology, Atlanta, Ga; and Pavilion Medical Innovations, ${ }^{\mathrm{c}}$ San Carlos, Calif.

Disclosures: Authors have nothing to disclose with regard to commercial support.

Received for publication Aug 29, 2011; revisions received Oct 20, 2011; accepted for publication Nov 9, 2011; available ahead of print Feb 17, 2012.

Address for reprints: Vinod H. Thourani, MD, Division of Cardiac Surgery, Department of Surgery, Emory University Hospital Midtown, 550 Peachtree Street, Sixth

Floor, MOT, Atlanta, GA 30308 (E-mail: vthoura@emory.edu).

$0022-5223 / \$ 36.00$

Copyright (C) 2012 by The American Association for Thoracic Surgery

doi:10.1016/j.jtcvs.2011.11.038 in front of the catheter or to assess the distance from the tip to an organ. To overcome this challenge, we adopted a novel cardioscopy system with a miniature camera at its tip, one that allows visualization of a volume immediately in front of the catheter. In the present study, we validated the use of this cardioscopy system in a porcine beating heart model.

\section{METHODS}

\section{Animal Model and Surgical Procedure}

Five $80-\mathrm{kg}$ Yorkshire pigs were used to conduct these studies. The procedure was performed through a median sternotomy in 4 pigs and by way of a minithoracotomy in 1 pig. All pigs were first premedicated with xylazine $(0.1 \mathrm{mg} / \mathrm{kg}$, intramuscularly), butorphanol $(0.2-0.4 \mathrm{mg} / \mathrm{kg}$, intramuscularly), and atropine $(0.1 \mathrm{mg} / \mathrm{kg}$, intramuscularly). After sedation, the pigs were taken to the preoperative staging area, and an intravenous line was placed and secured in the external jugular vein for induction with thiopental $(10 \mathrm{mg} / \mathrm{kg}$ ) intravenously, to effect. Thiopental was given slowly to avoid hypoglycemia and vasodilation. The pigs were intubated with the appropriate size endotracheal tube and mechanically ventilated. Anesthesia was maintained with continuous isoflurane inhalation $(1.5 \%-2 \%$ with oxygen), and the pigs were placed in the supine position on a heating blanket They were ventilated with oxygen-enriched room air at an initial setting of $10 \mathrm{breaths} / \mathrm{min}$ and $10 \mathrm{~mL} / \mathrm{kg}$ tidal volume. The ventilatory rates were adjusted to maintain a partial pressure of carbon dioxide of 30 to $40 \mathrm{~mm} \mathrm{Hg}$, partial pressure of oxygen greater than $100 \mathrm{~mm} \mathrm{Hg}$, and $\mathrm{pH}$ of 7.35 to 7.45 . 

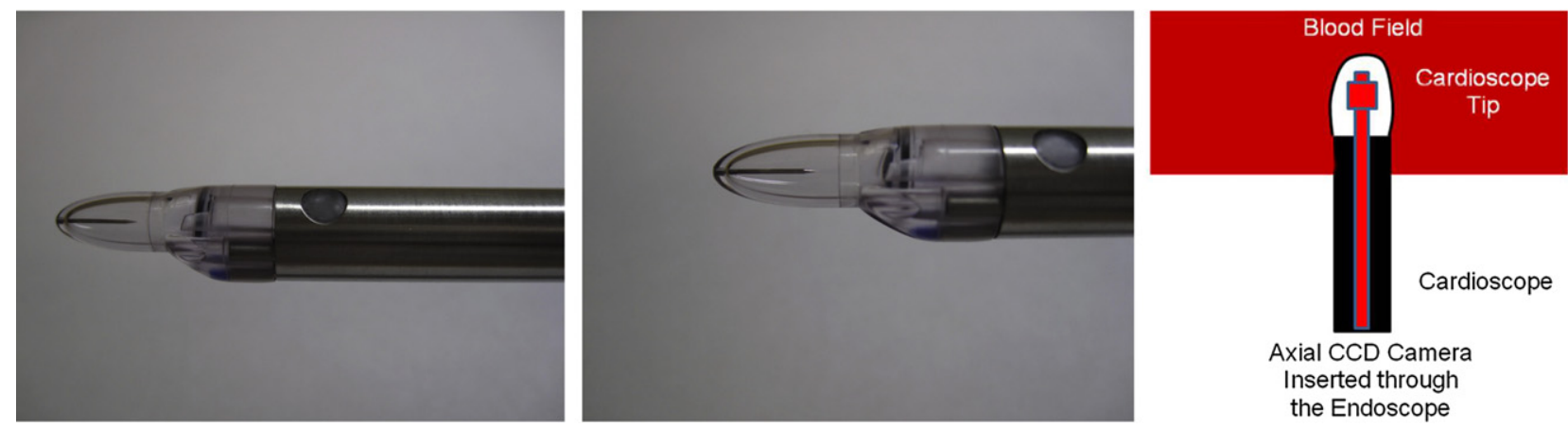

FIGURE 1. Schematic of the cardioscopy device used in the present study showing the blunt concave tip of the endoscope and the principle behind optical visualization through blood.

If unable to regulate acidemia by changing the ventilatory settings, acidemia was treated with sodium bicarbonate $(2-4 \mathrm{mEq} / \mathrm{kg}, 8.4 \%$ solution). The arterial blood gases were assessed at least every hour to ensure physiologic stability. Surface electrocardiographic leads were placed to provide continuous electrocardiographic data. A median sternotomy was performed in 4 of the pigs, and the heart was exposed by carefully separating the pericardium and exposing the apex. In 1 pig, a small left thoracotomy was performed to gain access to the apex of the heart, eliminating the need for a complete sternotomy.

\section{Cardioscopy}

In Figure 1, a schematic of the cardioscope system with the glass tip is depicted. In brief, the instrument is a typical 11-mm lumen endoscope with a nonblunt convex Plexiglass tip at the inserting end, and a CCD camera inside the tip for visualization. The size and shape of the tip is designed such that the curved Plexiglass shape displaces the tissue or blood in the immediate vicinity of the CCD camera, enabling focusing of the camera on the surrounding structures. The flexibility of the endoscope and a steering mechanism at its distal end allowed an excellent traversing angle of the tip after its insertion into the left ventricular cavity for good image acquisition of the valvular structures. The cardioscope was inserted into the ventricular cavity through a custom-designed port that allowed a bloodless field of operation. After introduction of the cardioscope into the left ventricle, the hemodynamics remained stable throughout the procedure. The cardioscope was manipulated within the left ventricular cavity, and the desired images were acquired of the ventricular, atrial, and valvular structures. Images of the beating mitral valve, chordae tendineae, and papillary muscles were acquired first, followed by directing the camera into the left atrium to identify the pulmonary vein insertion. The ventricular surface of the aortic valve leaflets was also recorded, after which the endoscope was advanced further into the ascending aorta to visualize the walls of the aorta and the main coronary arteries.

\section{RESULTS \\ Cardioscopy Implantation}

Cardioscopy was possible through the median sternotomy and minithoracotomy procedures. With the median sternotomy, direct access to the ventricular apex was possible (Figure 2, A). In the minithoracotomy case, a longer cannula-shaped system was used to achieve the same access through a 2-in. port (Figure 2,B).

\section{Mitral Valve Visualization}

Figure 3 shows a montage of the various components of the mitral valve imaged in 1 of the pigs, demonstrating the ability of the cardioscope system to image the papillary muscles, chordae tendineae, and mitral valve leaflets under beating heart conditions. Starting from the top left corner of the montage, Figure 3, A presents the view as the cardioscope is inserted through the apical port showing the walls of the device. Figure 3, $B$ shows the anterolateral papillary muscle of the mitral valve with the chordae tendineae originating from the muscle tip. The camera was then aligned parallel to the muscle tip, and the ventricular surface of the anterior leaflet was visualized with the web
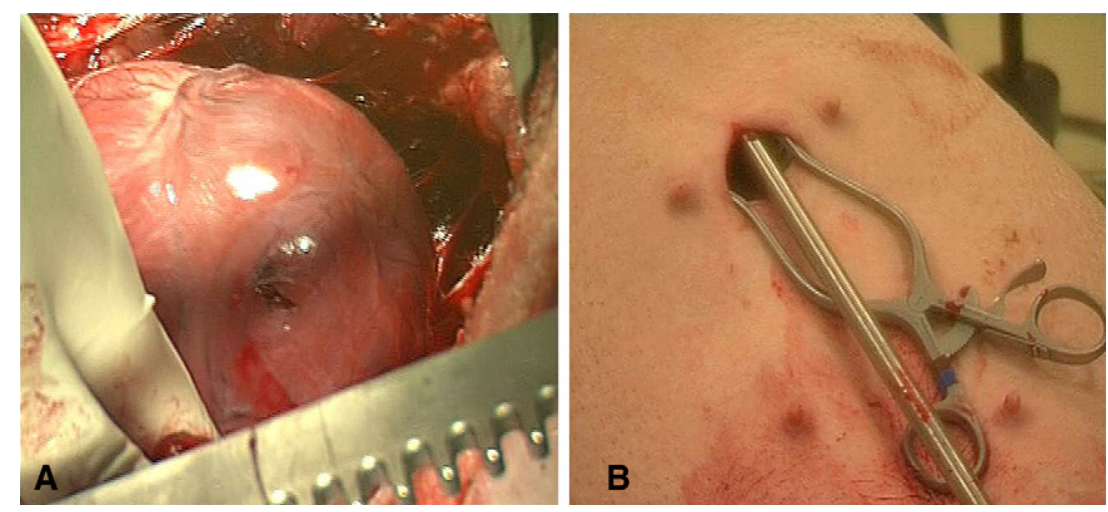

FIGURE 2. A and B, Apical access through a median sternotomy and the 2 -inch incision that was also tried in the present experimental study. 


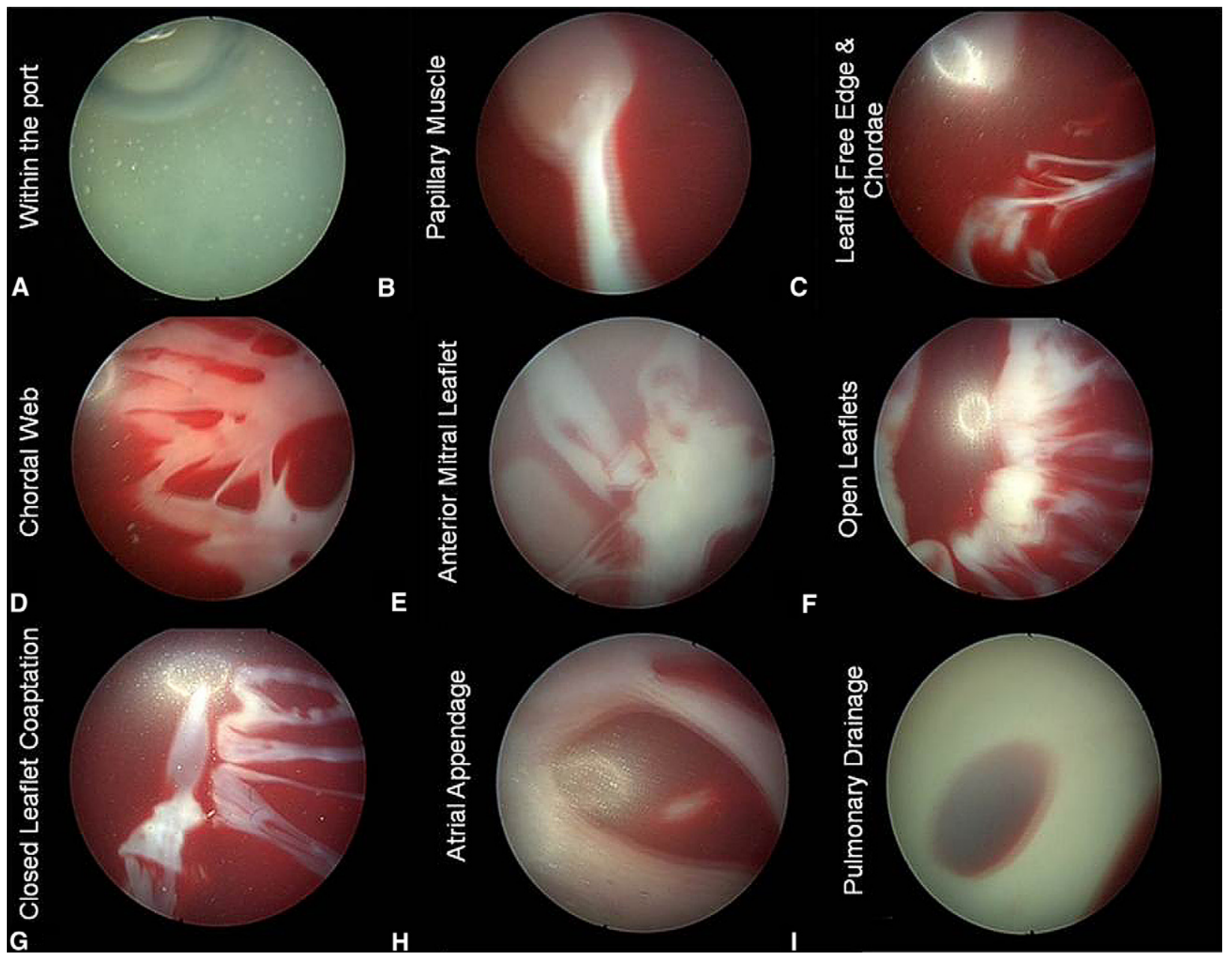

FIGURE 3. A-I, Montage of different images of the mitral valve obtained using the cardioscopy system depicting various components of the valve.

of chordae tendineae (Figure 3,C). As the cardioscope was maneuvered through the functional/beating valve, the web of marginal chordae and their insertion into the leaflets, was captured (Figure 3,D). In Figure 3, E, the atrial surface of the anterior leaflet is clearly evident, with the chordae tendineae inserting on the ventricular side of the leaflet. To obtain the closed and open positions of the mitral leaflets, the camera was positioned immediately below the coaptation zone in the left ventricle, and the diastolic orifice area (Figure 3, F) and the closed leaflet coaptation (Figure 3, G) were recorded. The cardioscope was then translated into the left atrium through the mitral valve, and the atrial appendage and pulmonary venous drainage into the left atrium were clearly evident (Figure 3, $H, I$ ).

\section{Aortic Valve Visualization}

The camera was then positioned within the left ventricle, and the ventricular surface of the aortic valve leaflets was imaged (Figure 4, A). Under direct optical visualization, the camera was passed into the proximal ascending aorta, and the left and right coronary ostia were visualized in the sinus of Valsalva (Figure 4, $B, C$ ). During this procedure, although transient mild aortic insufficiency was observed, it did not have a detrimental effect on the hemodynamics. Visualizing the aortic surface of the leaflets was not possible, owing to the limited traversing angle of the camera and the use of a nonrotatable rigid endoscopic end.

\section{DISCUSSION}

For the first time in published studies relevant to endoscopic cardiovascular surgery, we report the successful use of a transapical cardioscope to visualize the left heart structures under beating heart conditions. The present experimental study has demonstrated that beating heart endoscopic surgery inside the left ventricle is possible and alludes to the future possibilities in minimally invasive cardiothoracic surgery. The implications of this exciting technique on cardiothoracic surgery are numerous, but an immediate application of this technology is in heart valve repair and replacement. With increasing investment and enthusiasm for transcatheter aortic and mitral valve repair or 


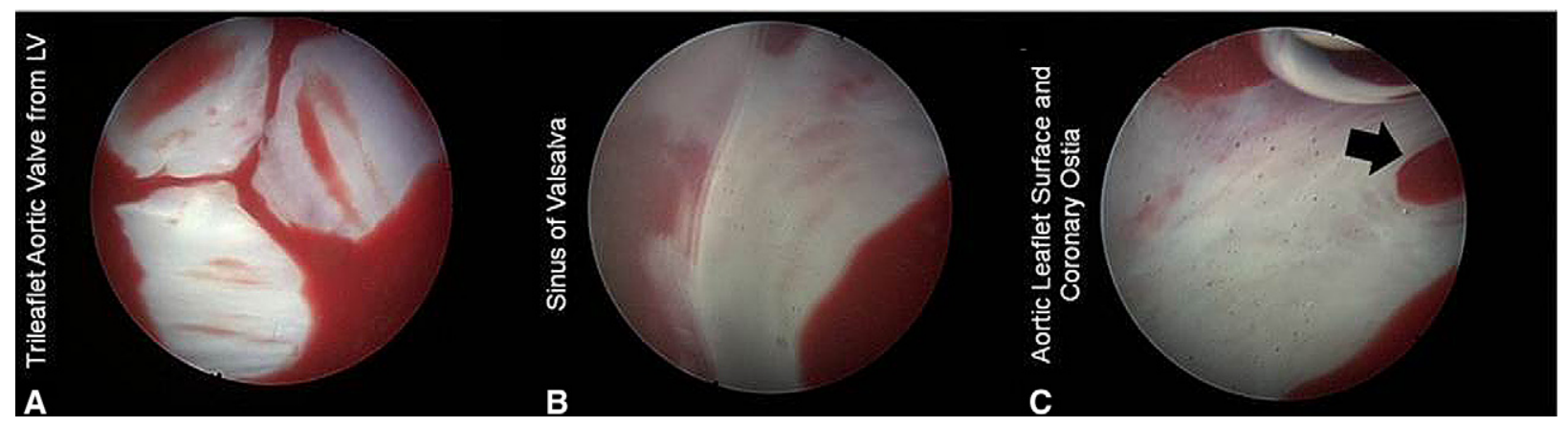

FIGURE 4. A-C, Montage of the ventricular surface of the aortic valve leaflets, the sinus of Valsalva, and the coronary ostia (arrow) as observed from the cardioscopy device under beating heart conditions.

replacement devices, a cardioscopy technique such as the one we have reported would be best suited to clearly assessing the pathologic features of the valve. Although 3-dimensional echocardiography and multislice, multidetector computed tomography has revolutionized the world of valve imaging, they do not and cannot substituted for direct visualization of the pathologic features (eg, a carcinoid tumor on the valve leaflets or leaflet distension and billowing in Barlow's disease). More specifically, this direct visualization might improve the positioning of transapical aortic valve replacement, leading to a decreased risk of valve misplacement and potentially reducing the incidence of paravalvular leak. In those transcatheter cases involving the mitral valve, this technique could improve the visualization of the defective leaflet segment and allow a more direct and expeditious therapeutic procedure. In those patients with a paravalvular leak after previous aortic or mitral valve surgery, this technique might improve the evaluation and treatment of transapical paravalvular leak repairs. Another potential application includes more direct and precise left atrial ablation for atrial fibrillation.

In addition to the pathologic diagnosis, an inherent limitation of 2- or 3-dimensional ultrasonography is that the plane of imaging is always perpendicular to the catheter axis. This is a significant limiting factor for procedures such as transapical access. For example, a transapical aortic valve sheath inserted into the left ventricular apex may have traversed the septum and reentered the left ventricular chamber; however, because the high-density sheath material would reflect more ultrasound energy than the tissue, a false image depicting no septal tissue overlying the catheter will be generated. With advances in fiberoptic technology, the cardioport system can be mounted on the sheath tip and provide live imaging of all the cardiac structures during the sheath translation. In addition to these applications, the use of the cardioport system in robotic surgery is tremendous. The CCD cameras can be mounted directly on the robotic arms or instruments or can be inserted through another smaller hole that provides a wide angle view of the surgical field. In essence, a clear clinical need exists for such a live imaging system, in addition to the current imaging modalities such as fluoroscopy and echocardiography.

In conclusion, we have introduced a first-generation endoscopic intraventricular optical visualization tool to visualize moving cardiac structures. Combined with preoperative imaging information, this tool may potentially open new avenues to perform cardiothoracic surgery. 